\title{
A Search for Thermal Vacancies Forming Close Frenkel Pairs in Fe-Cr Alloys
}

\author{
J. CHOJCAN \\ Institute of Experimental Physics, University of Wrocław \\ Pl. M. Borna 9, 50-204 Wrocław, Poland
}

\begin{abstract}
The room temperature Mössbauer spectra of ${ }^{57} \mathrm{Fe}$ were measured for the bcc $\mathrm{Fe}_{0.948} \mathrm{Cr}_{0.052}$ solid solution quenched into water being at about $295 \mathrm{~K}$ from different temperatures not exceeding $1000 \mathrm{~K}$, i.e. from the temperatures for which probabilities of formation of "clear" (Schottky) vacancies as well as vacancies forming the separate interstitial-vacancy (Frenkel) pairs are negligibly low. The obtained data were analysed in terms of concentration of unoccupied places in the 14-site surroundings of an ${ }^{57} \mathrm{Fe}$ Mössbauer probe in the sample. It turned out that the concentration of vacancies detected by the probe increases with temperature very rapidly - at the rate of about $10^{-4} \mathrm{~K}^{-1}$. The result may suggest that the Mössbauer probe is sensitive to a possible thermal distortion of the lattice or creation of very close Frenkel pairs (the interstitial is the nearest neighbour of the vacant lattice site).
\end{abstract}

PACS numbers: $61.72 . \mathrm{Ji}, 76.80 .+\mathrm{y}$

\section{Introduction}

It is commonly thought that thermally formed vacancies in metals are "clear" (Schottky) vacancies [1] as they are directly observed with differential dilatometry [2] and from the theoretical studies it follows that formation enthalpy of vacancies forming separate interstitial-vacancy (Frenkel) pairs is essentially larger than that for the Schottky vacancies [3]. In the case of pure iron the formation enthalpy for "clear" vacancies amounts to $1.7 \mathrm{eV}$ and concentration of the thermal vacancies above $1070 \mathrm{~K}$ is high enough to be detected by positrons [4], the most sensitive probes to the defects beside positive muons [1]. However our recent findings $[5,6]$ concerning Fe-Cr alloys quenched from different temperatures suggest that the ${ }^{57} \mathrm{Fe}$ Mössbauer spectroscopy can be successfully applied to the study of both Schottky and Frenkel thermal vacancies; forming maybe a kind of close interstitial-vacancy pairs [1] in the latter case. Such conclusion can be reached if 
it is noticed that for the $\mathrm{Fe}-\mathrm{Cr}$ systems being at temperatures higher than about $1000 \mathrm{~K}$, the concentration of vacancies detected by the ${ }^{57} \mathrm{Fe}$ probe in its neighbourhood is enormous (0.01-0.1), at least one order of magnitude greater than that expected for clear Schottky vacancies. The latter can be understood taking into account the theoretical calculations of lattice distortion around individual point defects having the symmetry of the lattice. The calculations show that the second neighbours of the defects are relaxed in a direction opposite to that of the first neighbours and in the case of a vacancy the first neighbours are displaced inwards and the second neighbours outwards [7]. In the other words, formation of a vacancy having the symmetry of the lattice (e.g. the Schottky vacancy) goes together with the creation of several additional vacancies not having the symmetry mentioned and forming a kind of close Frenkel pairs (very close) with atoms displaced from their early positions due to introduction of the symmetric vacancy into a crystal. Accepting the theoretical findings one can say that concentration of the very close Frenkel pairs (the interstitial is the nearest neighbour of the vacancy) could be up to about one order of magnitude greater than the symmetric vacancies.

In the present work the exceptional sensitivity of the ${ }^{57} \mathrm{Fe}$ Mössbauer probe to any vacancy located in its neighbourhood is used for investigation of quenched-in vacancies in several samples of $\mathrm{Fe}-\mathrm{Cr}$ solid solution containing about 5 at.\% of chromium and being quenched from different temperatures at which the concentration of the Schottky vacancies should be negligibly low, i.e. temperatures not exceeding $1000 \mathrm{~K}$.

\section{Experimental and results}

The iron-chromium alloy was prepared (see [8] for details) by melting the Armco iron and electrolytic chromium of $99.99 \%$ purity in a vacuum induction furnace. The obtained ingot was forged and cold-rolled to the final thickness of about $0.06 \mathrm{~mm}$ and then the foil was cut into several similar pieces. Next, each sample was annealed in vacuum at about $1300 \mathrm{~K}$ for $0.5 \mathrm{~h}$ and slowly furnace cooled to room temperature for $6 \mathrm{~h}$. Under these conditions, diffusion effectively stops at about $700 \mathrm{~K}$ [9], so that the observed distributions of atoms in the annealed specimens should be the frozen-in state corresponding to $700 \mathrm{~K}$ whereas concentration of vacancies (practically zero) should be determined by the room temperature. The composition of the samples was controlled with an analysis of the energy distribution of X-ray (EDX) induced by the $30 \mathrm{keV}$ electron beam. The EDX data showed that the specimens studied contained $5.2 \pm 0.2$ at.\% of chromium. Next, the annealed samples heated in an argon atmosphere at different temperatures $T$ not exceeding $1000 \mathrm{~K}$, were quenched in water being at about $295 \mathrm{~K}$ in order to freeze the high temperature vacancies as well as distribution of atoms in the specimens. After the quenching process each sample was chemically polished to remove possible surface contaminants. 
The measurements of the ${ }^{57} \mathrm{Fe}$ Mössbauer spectra were performed in transmission geometry by means of a constant-acceleration POLON spectrometer of standard design. The $14.4 \mathrm{keV}$ gamma rays were provided by a $1.4 \times 10^{9} \mathrm{~Bq}$ source of ${ }^{57} \mathrm{Co}$ dissolved in rhodium. The spectra were measured at room temperature using 512-channel analyser. The number of counts per channel and the spectrum maximum depth were $(3-4) \times 10^{6}$ and $(2-3) \times 10^{5}$, respectively. Some of the spectra are presented in Fig. 1.

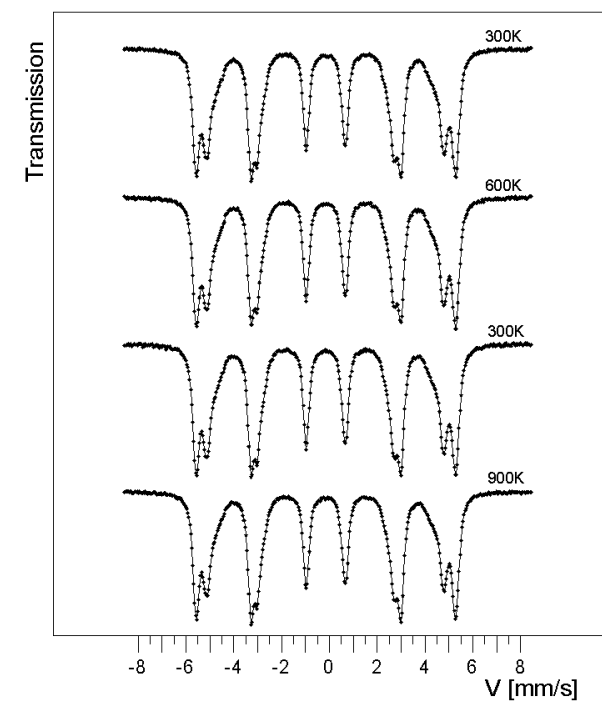

Fig. 1. The Mössbauer spectra of ${ }^{57} \mathrm{Fe}$ for the $\mathrm{Fe}_{0.948} \mathrm{Cr}_{0.052}$ alloy annealed and quenched (sample no. 3). The spectra of the annealed and slowly cooled sample from $1300 \mathrm{~K}$ to the room temperature are marked with $300 \mathrm{~K}$.

All spectra were analysed in terms of four six-line patterns corresponding to different hyperfine fields $(\mathrm{HF})$ at ${ }^{57} \mathrm{Fe}$ nuclei determined by numbers of $\mathrm{Fe}$ and $\mathrm{Cr}$ atoms located in the vicinity of the nuclei, assuming that the effect of a $\mathrm{Cr}$ atom on $\mathrm{HF}$ is independent of its position in the surroundings of the probe. Moreover, it was accepted that the field (and the corresponding centre shift of the subspectrum) is a linear function of the number $n$ of $\mathrm{Cr}$ neighbours of ${ }^{57} \mathrm{Fe}$ with the form: $\mathrm{HF}_{n}=\mathrm{HF}_{0}+n \Delta \mathrm{HF}$, where $\Delta \mathrm{HF}$ stands for the change of $\mathrm{HF}$ with one $\mathrm{Cr}$ atom in the neighbourhood of the Mössbauer probe. Finally, it was assumed that the three line widths $\Gamma_{16}, \Gamma_{25}$, and $\Gamma_{34}$ as well as the two line intensity ratios $I_{16} / I_{34}$ and $I_{25} / I_{34}$ are the same for all six-line components of the given spectrum. As a result of the Mössbauer spectrum analysis the intensities $c_{\mathrm{Cr}}^{\prime}$ and $c_{\mathrm{CrCr}}^{\prime}$ of the second and third components of each spectrum were determined. The components are related to the existence of one $\mathrm{Cr}$ atom and two $\mathrm{Cr}$ atoms in the neighbourhood of the Mössbauer probe, respectively. The results are listed in Table. 
TABLE

The binding energy between a pair of $\mathrm{Cr}$ atoms $\left(E_{\mathrm{b}}^{\prime}\right)$ and concentration of vacancies $\left(c_{\mathrm{V}}\right)$, deduced from the Mössbauer spectra of ${ }^{57} \mathrm{Fe}$ in the $\mathrm{Fe}_{0.948} \mathrm{Cr}_{0.052}$ alloy annealed at about $1300 \mathrm{~K}$ and then quenched from different temperatures. The standard deviations $\sigma$ for $c_{\mathrm{Cr}}^{\prime}$ and $c_{\mathrm{CrCr}}^{\prime}$ result from the variance of the fit of the assumed model to the spectrum measured. The $\sigma$ values for $c_{V}$ were computed taking into account the $c_{\mathrm{Cr}}^{\prime}$ and $c_{\mathrm{CrCr}}^{\prime}$ errors only.

\begin{tabular}{c|c|c|c|c|l}
\hline \hline Sample no. & $T[\mathrm{~K}]$ & $c_{\mathrm{Cr}}^{\prime}$ & $c_{\mathrm{CrCr}}^{\prime}$ & $E_{\mathrm{b}}^{\prime}[\mathrm{meV}]$ & \multicolumn{1}{c}{$c_{\mathrm{V}}$} \\
\hline \multirow{4}{*}{1} & 300 & $0.398(2)$ & $0.109(1)$ & $22(2)$ & 0 \\
& 400 & $0.397(2)$ & $0.108(1)$ & & $0.006(3)$ \\
& 300 & $0.398(2)$ & $0.110(1)$ & $21(2)$ & 0 \\
& 700 & $0.393(2)$ & $0.104(1)$ & & $0.038(3)$ \\
& 300 & $0.400(2)$ & $0.108(1)$ & $23(2)$ & 0 \\
& 500 & $0.398(2)$ & $0.109(1)$ & & $-0.012(3)$ \\
& 300 & $0.396(2)$ & $0.110(1)$ & $21(2)$ & 0 \\
& 800 & $0.392(2)$ & $0.102(1)$ & & $0.083(3)$ \\
& 300 & $0.396(2)$ & $0.109(1)$ & $22(2)$ & 0 \\
& 600 & $0.399(2)$ & $0.106(1)$ & & $0.031(3)$ \\
& 300 & $0.400(2)$ & $0.110(1)$ & $22(2)$ & 0 \\
& 900 & $0.383(2)$ & $0.106(1)$ & & $0.045(3)$ \\
& 300 & $0.396(2)$ & $0.105(1)$ & $25(2)$ & 0 \\
& 1000 & $0.377(1)$ & $0.104(1)$ & & $0.045(3)$
\end{tabular}

The $c_{\mathrm{Cr}}^{\prime}$ and $c_{\mathrm{CrCr}}^{\prime}$ values for annealed samples were used for calculating the binding energy $E_{\mathrm{b}}^{\prime}$ for pairs of Cr atoms in the studied materials. The computations were performed, as in [10], on the basis of the modified Hrynkiewicz-Królas formula $[11,12]$, assuming that the ratio of the concentrations of the individual pairs of $\mathrm{Cr}$ atoms and isolated $\mathrm{Cr}$ atoms in the specimen is equal to the quotient of the intensities $c_{\mathrm{CrCr}}^{\prime}$ and $c_{\mathrm{Cr}}^{\prime}$ mentioned above

$$
\begin{aligned}
E_{\mathrm{b}}^{\prime}= & -k T_{\mathrm{d}} \\
& \times \ln \left[\left(1+2 c_{\mathrm{CrCr}}^{\prime} / c_{\mathrm{Cr}}^{\prime}\right)\left(c_{\mathrm{CrCr}}^{\prime} / c_{\mathrm{Cr}}^{\prime}\right)(1+2 p(2) / p(1))^{-1}(p(2) / p(1))^{-1}\right],
\end{aligned}
$$

where $k$ is the Boltzmann constant, $T_{\mathrm{d}}$ denotes the "freezing" temperature for the atomic distribution in the sample (due to the slow cooling of the annealed samples $\left.T_{\mathrm{d}}=700 \mathrm{~K}\right), p(n)=[N ! /((N-n) ! n !)] x^{n}(1-x)^{N-n}$ is the probability for the existence of $n \mathrm{Cr}$ atoms in the $N$-atom neighbourhood of the Fe atom (the formula results from statistical considerations for random distributions of two kinds of non-interacting atoms in a given lattice) so $p(2) / p(1)=0.5 x(N-1) /(1-x)$, $x$ stands for the concentration of $\mathrm{Cr}$ atoms and $N=8+6=14$ (the total number of the lattice sites in the two first coordination shells of an atom in the bcc lattice 


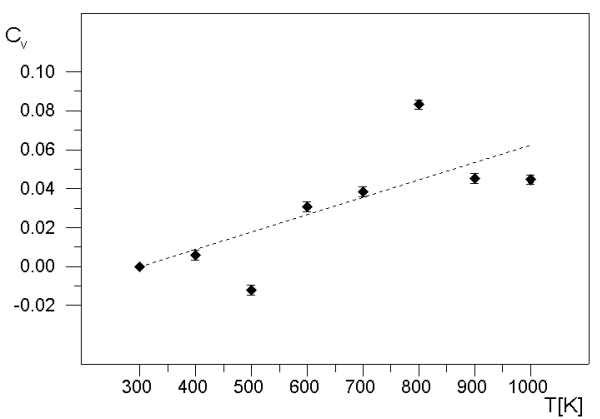

Fig. 2. Concentration $c_{V}$ of vacancies detected by the ${ }^{57} \mathrm{Fe}$ Mössbauer probes in the bcc $\mathrm{Fe}_{0.948} \mathrm{Cr}_{0.052}$ samples as a function of the temperature from which the sample was quenched. The dashed line is a plot of the function $f(T)=\beta+\alpha T$, fitted to the $c_{\mathrm{V}}$ values by the least squares method; $\alpha=(0.9 \pm 0.2) \times 10^{-4} \mathrm{~K}^{-1}$.

- compare [13, 8] for example). The $E_{\mathrm{b}}^{\prime}$ values obtained for annealed samples are collected in Table. The values were used to estimate the effective number $N$ of nearest neighbours having a direct influence on the Mössbauer probe in the quenched specimens. It was done accepting that the values of $E_{\mathrm{b}}^{\prime}$ are temperature independent and applying Eq. (1) in the more convenient form

$$
\begin{aligned}
N= & 0.5\{3 x-1+(1-x) \\
& \left.\times\left[1+8\left(1+2 c_{\mathrm{CrCr}}^{\prime} / c_{\mathrm{Cr}}^{\prime}\right)\left(c_{\mathrm{CrCr}}^{\prime} / c_{\mathrm{Cr}}^{\prime}\right) \exp \left(+E_{\mathrm{b}}^{\prime} /\left(\mathrm{kT}_{\mathrm{d}}\right)\right)\right]^{0.5}\right\} / \chi .
\end{aligned}
$$

Finally, for the quenched samples we determined the concentration $c_{\mathrm{V}}=$ $(14-N) / 14$ of vacancies or unoccupied places in the 14-site neighbourhood of an ${ }^{57} \mathrm{Fe}$ probe atom. The results are presented in Table and Fig. 2.

\section{Conclusions}

The data obtained (Table) support our early findings [10] that the nature of the $\mathrm{Cr}$ pair interaction in the iron crystal is repulsive and the binding energy $E_{\mathrm{b}}^{\prime}$ between two $\mathrm{Cr}$ atoms in the $\mathrm{Fe}-\mathrm{Cr}$ system containing about 5 at.\% of chromium is close to $20 \mathrm{meV}$. The obtained $E_{\mathrm{b}}^{\prime}$ value is greater than that resulting from the semi-empirical Miedema-Królas model [14, 15] and predicted for Fe with trace concentration of $\mathrm{Cr}$; the latter is $15 \mathrm{meV}$. This may suggest that the $E_{\mathrm{b}}^{\prime}$ value resulting from the Miedema-Królas model is underestimated.

The concentration $c_{V}$ of quenched-in vacancies detected by the ${ }^{57} \mathrm{Fe}$ Mössbauer probe in its neighbourhood for the bcc $\mathrm{Fe}_{0.948} \mathrm{Cr}_{0.052}$ solid solution strongly depends on the quenching temperature. The slope $\alpha$ of the straight line fitted to the $c_{\mathrm{V}}$ data (see Fig. 2) amounts to $(0.9 \pm 0.2) \times 10^{-4} \mathrm{~K}^{-1}$. The result is in agreement with our positron annihilation measurements for the materials studied. The measurements show that positron lifetime $\tau$ in the $\mathrm{Fe}-\mathrm{Cr}$ specimens increases with the quenching temperature (see Fig. 3) which suggests that an increase in 


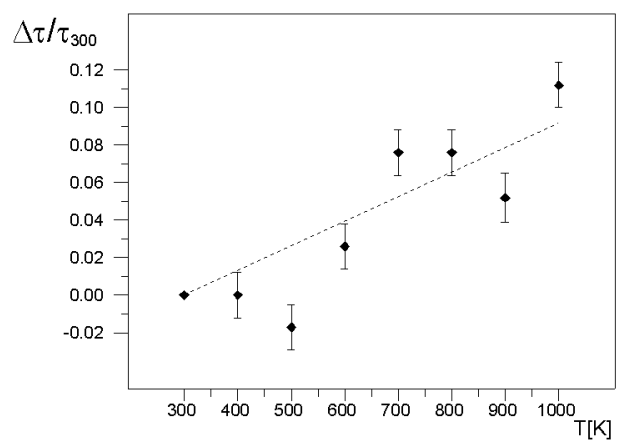

Fig. 3. The mean lifetime $\tau$ of positrons in the bcc $\mathrm{Fe}_{0.948} \mathrm{Cr}_{0.052}$ samples as a function of the temperature from which the sample was quenched. $\Delta \tau=\tau-\tau_{300}$, where $\tau_{300}$ denotes $\tau$ for the temperature $300 \mathrm{~K}$. The dashed line is a plot of the function $f(T)=\beta+\alpha T$, fitted to the $\Delta \tau / \tau_{300}$ values by the least squares method; $\alpha=(1.3 \pm 0.2) \times 10^{-4} \mathrm{~K}^{-1}$.

the temperature goes together with creation of some low-electron-density regions in the sample that attract positrons. Moreover, the rate $\alpha$ of the temperature increase in $\tau$ amounts to $(1.3 \pm 0.2) \times 10^{-4} \mathrm{~K}^{-1}$ (see Fig. 3) and it is almost the same as for $c_{\mathrm{V}}$. Taking the above into account one can suppose that most of the low-electron-density regions observed by positrons are the vacancies detected by ${ }^{57} \mathrm{Fe}$ Mössbauer nuclei. Because the applied temperatures are relatively low, the probes under consideration can "see" neither Schottky vacancies nor vacancies forming the separate Frenkel pairs. In these circumstances it seems reasonable to conclude that the Mössbauer probes are sensitive to small thermal distortions of the lattice which are observed by the probes as very close Frenkel pairs (the interstitial is the nearest neighbour of the vacant lattice site). On the other hand, one should remember that the conclusion is not supported by the theoretical computations from which it follows that in pure iron all individual close Frenkel pairs are unstable against recombination [16]. Bearing this in mind, it is obvious that proper explanation of the obtained results needs further experimental and theoretical studies.

\section{Acknowledgments}

This work was supported by the University of Wrocław under the grant 2016/IFD/2001. 


\section{References}

[1] A. Seeger, Phys. Status Solidi A 167, 289 (1998).

[2] K. Mosig, J. Wolff, J.-E. Kluin, Th. Hehenkamp, J. Phys., Condens. Matter 4, 1447 (1992).

[3] R. Bauer, W. Maysenhölder, A. Seeger, Phys. Lett. A 90, 55 (1982).

[4] H.-E. Schaefer, Phys. Status Solidi A 102, 47 (1987).

[5] J. Chojcan, J. Beliczyński, Mol. Phys. Rep. 22, 21 (1998).

[6] J. Chojcan, J. Beliczyński, Mol. Phys. Rep. 30, 27 (2000).

[7] J. Takamura, in: Physical Metallurgy, Ed. R.W. Cahn, North-Holland, Amsterdam 1965, p. 682.

[8] S.M. Dubiel, J. Żukrowski, J. Korecki, K. Krop, Acta Phys. Pol. A 47, 199 (1975).

[9] T.E. Cranshaw, J. Phys., Condens. Matter 1, 829 (1989).

[10] J. Chojcan, Phys. Status Solidi B 219, 375 (2000).

[11] A.Z. Hrynkiewicz, K. Królas, Phys. Rev. B 28, 1864 (1983).

[12] J. Chojcan, J. Alloys Comp. 264, 50 (1998).

[13] I. Vincze, I.A. Campell, J. Phys. F 3, 647 (1973).

[14] A.R. Miedema, Physica B 182, 1 (1992).

[15] K. Królas, Phys. Lett. A 85, 107 (1981).

[16] C. Erginsoy, G.H. Vineyard, A. Englert, Phys. Rev. 133, A595 (1964). 